$\pm \mathrm{CABG}$ was 73.8 years, whilst average age for patients who had TAVI was 83.9 years, otherwise average age for valvuloplasty and medical treatment groups were 83.6 and 85.6 years respectively.

Conclusion Almost 3 out of each 4 patients with severe AS followed up in a tertiary cardiac centre were symptomatic, and more than two thirds of them underwent intervention. On average patients who had surgical AVR \pm CABG were almost a decade younger than patients in other treatment groups including patients who had TAVI, valvuloplasty and medical treatment. Most of the patients with asymptomatic severe AS were managed conservatively. This study highlights the high prevalence of symptoms within this population, and has proven useful to estimate the number of annual procedures required based on the number of symptomatic patients. Conflict of Interest None

\section{COMMUNITY-ACQUIRED CULTURE-NEGATIVE ENDOCARDITIS: CLINICAL CHARACTERISTICS AND OUTCOMES}

Anass Maaroufi, Hatim Zahidi, Marwa Abdulhakeem, Rachida Habbal. Ibn Rochd University Hospital, Casablanca, Morocco

10.1136/heartjnl-2021-BCS.31

Introduction Community-acquired culture-negative endocarditis is a rare entity poorly studied.

Objectives We studied the clinical characteristics, in-hospital mortality, and short-term prognosis of patients with culturenegative endocarditis.

Methods In total, 104 episodes of definite endocarditis according to duke criteria, were studied (2014-2019). We compared the clinical, laboratory, and echocardiography characteristics and the complications and survival rates of patients with culture-negative and culture-positive endocarditis.

Results Culture-negative endocarditis occurred in 76/104 (73\%) episodes. Compared with the culture-positive endocarditis patients, the time elapsed between first symptoms and admission was lower in patients with culture-negative endocarditis, a mean of 20 days vs 30 days in culture positive group $(\mathrm{p}<0.05)$, and these patients also had lower C-reactive protein levels at admission (99 vs $120 \mathrm{mg} / \mathrm{dl}$ ) ( $<<0.05$ ). in hospital stay was not diffirent between the two groups with a mean of days of hospitalization of 27 days . However in-hospital mortality rates were higher in culture-negative versus culture-positive patients ( $15 \%$ vs $11 \%$ mortality rate) . as for comlication rates Severe sepsis and vascular comlication (stroke , splenic infarction) were higher in the negative culture group with $7.9 \%$ vs $3.1 \%$ in the positive culture groupe $\mathrm{p}<0.05$.

Conclusions Culture-negative endocarditis patients presented with lower levels of C-reactive protein at admission and required less time for hospital admission, however presented a higher rate of in-hospital mortality and complications compared to culture-positive endocarditis patients.

Conflict of Interest none

\section{INFECTIVE ENDOCARDITIS: A REVIEW OF THE PATIENT JOURNEY AT A DISTRICT GENERAL HOSPITAL (DGH)}

Leanne Eveson, Heather Nicholson, Amani Patel, Catherine MacFarlane, Ann Parker, David Garner, Lydia Sturridge. Frimley Park Hospital, Frimley, UK
Introduction Infective endocarditis (IE) is a rare and potentially fatal infection. Patients often present with generalised symptoms resulting in delays in diagnosis. Complications are common and patients can have long inpatient stays due to intravenous antibiotic requirement. The POET study highlighted non inferiority of oral antibiotic switch in stable patients with left sided IE. The aim of our study is to characterise the IE patient population at our $\mathrm{DGH}$, analyse their management, review adherence of our Endocarditis Team to the ESC 2015 guidelines (figure 1) and to review discharge information and advice, to identify areas for improvement.

Methods This was a retrospective study of medical records for patients treated at our DGH with a diagnosis of IE between 1 Oct 2019 and 30 Sep 2020. Demographics, patient characteristics (table 1), IE risk factors, presentation, management, discharge information, patient feedback, and cost analysis was carried out. Adherence of our Endocarditis Team to ESC 2015 guidelines was reviewed.

Results Between October 2019 and September 2020, 14 patients were diagnosed with IE according to the Duke Criteria. The median age was $75(60-89)$ years and $68.7 \%$ of patients were male. 7 patients had a prosthetic valve and 3 had a cardiac device in situ. All patients had CVR risk factors, $50 \%$ had T2DM, 36\% had renal disease. Only 2 patients had documentation of dental history. The majority of patients $(n=12)$ presented via the Emergency Department. $86 \%$ of patients completed a 6 -week antibiotic course. Only $14 \%$ of patients were managed as outpatients. $21 \%$ of patients required surgical management. All patients were reviewed by the Endocarditis Team. All patients were followed up appropriately. Only 1 patient had documented advice regarding dental care. 1 patient died and many patients had complications (figure 2). IE patient support group feedback highlighted concerns regarding delay in diagnosis, challenges of a long inpatient stay and benefits of hearing from other patients about their experiences.

\section{Abstract 32 Table 1 Patient characteristics}

Peripheral stigmata of IE

Echocardiographic evidence of vegetation

Role of the 'Endocarditis Team'

I. The 'Endocarditis Team' should have meetings on a regular basis in order to discuss cases, take surgical decisions, and define the type of follow-up.

2. The 'Endocarditis Team' chooses the type, duration, and mode of follow up of antibiotic therapy, according to a standardized protocol, following the current guidelines.

3. The 'Endocarditis Team' should participate in national or international registries, publicly report the mortality and morbidity of their centre, and be involved in a quality improvement programme, as well as in a patient education programme.

4. The follow-up should be organized on an outpatient visit basis at a frequency depending on the patient's clinical status (ideally at I, 3, 6 , and 12 months after hospital discharge, since the majority of events occur during this period ${ }^{57}$ ).

Abstract 32 Figure 1 


\section{Complications}

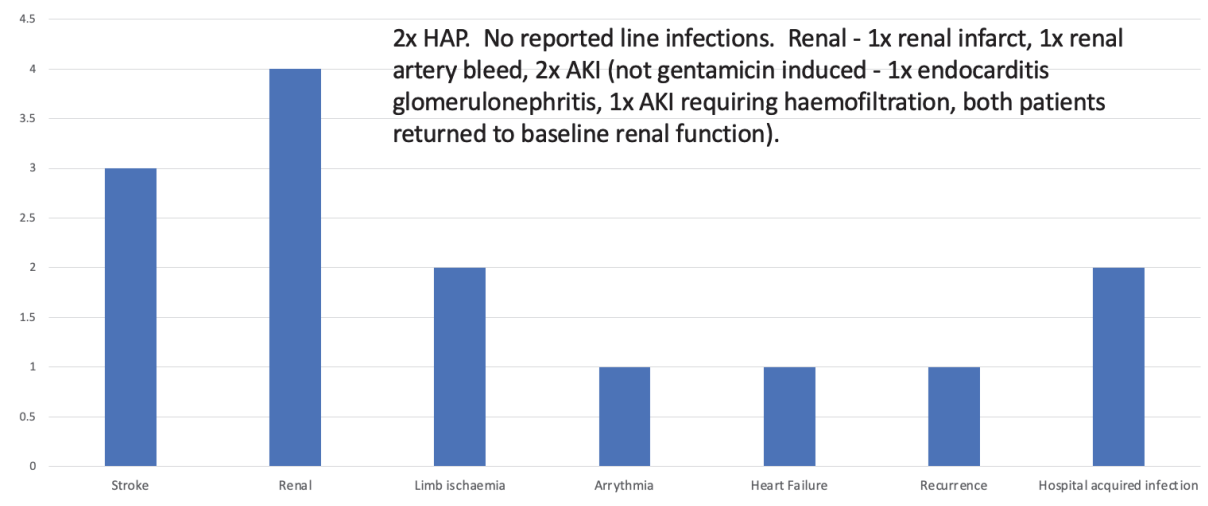

\section{Abstract 32 Figure 2}

Conclusion IE is a rare disease. Documentation of some IE risk factors, and documented discharge advice was poor at our centre. Our Endocarditis Team meet weekly, have a significant input in IE patient management and ensure adequate follow up is arranged for patients. We plan to join an international IE registry. An IE ward round proforma has been created along with a teaching session for staff to improve awareness and understanding of IE. A discharge information pack including information on IE, dental advice and IE and dental warning cards has been created. We plan for a virtual IE support group given current COVID-19 restrictions. We plan to create an outpatient IE treatment pathway with potential for improvement in patient experience and potential for significant cost savings.

Conflict of Interest None

\section{Acute coronary syndromes \& Interventional cardiology}

\section{ABSOLUTE CORONARY BLOOD FLOW AND MICROVASCULAR RESISTANCE MODELLING IN PATIENTS WITH CORONARY ARTERY DISEASE}

\footnotetext{
'Louise Aubiniere-Robb, ${ }^{2}$ Paul Morris, ${ }^{2}$ Julian Gunn, ${ }^{2}$ Rebecca Gosling, ${ }^{3}$ Rodney Hose, ${ }^{3}$ Vignesh Rammohan, ${ }^{3}$ Patricia Lawford. ${ }^{1}$ NHS Greater Glasgow and Clyde, Glasgow, UK; ${ }^{2}$ Sheffield Teaching Hospitals; ${ }^{3}$ University of Sheffield
}

\subsection{6/heartjnl-2021-BCS.33}

Background Ischaemic heart disease causes reduced coronary blood flow $(\mathrm{CBF})$. CBF is not routinely measured in the catheterisation laboratory. Cardiologists therefore rely upon surrogate indices of CBF like fractional flow reserve (FFR). Whilst valuable in guiding revascularisation decisions, FFR reports $\mathrm{CBF}$ as a fraction of a hypothetical and unknown value. It is therefore, semi-quantitative and cannot assess microvascular physiology. FFR-guided PCI is associated with incomplete symptom resolution in around $20 \%$ of patients. These limitations may be resolved by a new method (virtuQ ${ }^{\mathrm{TM}}$ ) which computes absolute coronary blood flow (aCBF) and coronary microvascular resistance (CMVR) from angiography and standard pressure wire measurements. The aims of our study were to establish the relationship between FFR and aCBF, and to investigate the contribution of CMVR to aCBF.

Methods virtuQ ${ }^{\mathrm{TM}}$ software was used to reconstruct the 3-D arterial anatomy of 229 vessels from 151 patients undergoing angiography and FFR assessment for coronary syndromes. $\mathrm{aCBF}$ and CMVR were computed by a numerical method based upon the reconstructed anatomy, pressure conditions and Navier-Stokes equations. The reduction in aCBF due to epicardial disease was also calculated. Percentage flow reduction (predicted by FFR) was compared to aCBF reduction in $\mathrm{mL} / \mathrm{min}$. A threshold for intervention for aCBF reduction was derived. Agreement between FFR and aCBF reduction was assessed by Cohen's kappa ( $\kappa)$ statistic.

Results virtuQ ${ }^{\mathrm{TM}}$ computed all physiological parameters in 207/ 229 cases (90\%). Physiological results are summarised in table 1. Calculated by regression intercept using an FFR-aCBF plot, the derived threshold for physiological significance for aCBF reduction was $\geq 23 \mathrm{~mL} / \mathrm{min}$ (figure 1). Overall agreement between FFR and aCBF reduction was moderate $(\kappa=0.70)$. Agreement between FFR and aCBF reduction was high in cases where FFR was $>0.80$ (90.0\%) and perfect when FFR $\leq 0.70$ (100\%), but poor when FFR was $0.70-0.80$ (68.2\%). For cases in which FFR was $\leq 0.80(n=109), 19.5 \%$ were discordant, associated with increased CMVR (1.24 vs 0.58 mmHg.min/mL, P<0.0001). A hybrid assessment strategy (FFR alone if $>0.80$ or $\leq 0.70$, with aCBF used if FFR $0.70-0.80$ )

\begin{tabular}{|c|c|c|c|c|}
\hline $\begin{array}{l}\text { Physiological } \\
\text { parameter } \\
\text { (Median) }\end{array}$ & $\begin{array}{l}\text { All } \\
\text { cases } \\
(n=207)\end{array}$ & $\begin{array}{l}\text { Females } \\
(\mathrm{n}=45)\end{array}$ & $\begin{array}{l}\text { FFR positive } \\
(\mathrm{n}=113)\end{array}$ & $\begin{array}{l}\text { FFR negative } \\
(n=94)\end{array}$ \\
\hline Baseline aCBF (mL/min) & 57.4 & 55.0 & 55.9 & 57.9 \\
\hline $\begin{array}{l}\text { Hyperaemic aCBF (mL/ } \\
\mathrm{min})\end{array}$ & 85.2 & 79.5 & 92.3 & 82.3 \\
\hline aCBF Reduction (mL/min) & 21.1 & 21.1 & 34.6 & 12.0 \\
\hline Baseline CMVR & 1.28 & 1.59 & 1.24 & 1.33 \\
\hline Hyperaemic CMVR & 0.72 & 0.86 & 0.65 & 0.80 \\
\hline
\end{tabular}

